\title{
Metaheuristic Global Path Planning Algorithm for Mobile Robots
}

\author{
Stojanche Panov \\ Faculty of Computer Science, \\ "Goce Delcev" - University, Stip, Macedonia \\ E-mail: stojance.panov@ugd.edu.mk \\ Saso Koceski* \\ Faculty of Computer Science, \\ "Goce Delcev" - University, Stip, Macedonia \\ E-mail: saso.koceski@ugd.edu.mk \\ ${ }^{*}$ Corresponding author
}

\begin{abstract}
A new metaheuristic method applied to the global path planning for mobile robots in dynamic environments is presented. This algorithm, named the Quad Harmony Search method, consists of dividing the robot's environment into free regions by applying the Quad-tree algorithm and utilizing this information to accelerate the next phase which implements the Harmony Search optimization method to provide the optimal route. The presented results have displayed that this method gives best results when compared to other metaheuristic techniques and is therefore applicable to the global path planning problem.
\end{abstract}

Keywords: dynamic environments, free space decomposition, global path planning, metaheuristic algorithms, optimization.

Reference to this paper should be made as follows: Panov, S. and Koceski, S. (2014) 'Metaheuristic Global Path Planning Algorithm for Mobile Robots', Int. J. High Performance Computing and Networking, Vol. 6, Nos. 2, pp.43-54.

Biographical notes: Stojanche Panov obtained his Master's degree in robotics and artificial intelligence in 2013 at the Faculty of Computer Science, "Goce Delchev" University - Stip. His main research interests are robotics, artificial intelligence algorithms, cryptography and game theory.

Saso Koceski is an assistant professor at the Faculty of Computer Science, University "Goce Delcev"-Stip, Macedonia and head of the Institute of Computer Science. He serves as an editor and reviewer in several SCI journals. His current research interests are focused in the field of robotics and artificial intelligence, bioinformatics, HCI and medical imaging.

This paper is a revised and expanded version of a paper entitled 'Global Path Planning Algorithm for Mobile Robots' presented at XLVIII International Scientific Conference on Information, Communication and Energy Systems and Technologies, ICEST 2013, 26-29 June 2013, Ohrid, Republic of Macedonia.

\section{INTRODUCTION}

The main definition of the global path planning problem would be explained as providing a feasible or optimal route given initial and destination point in a predefined environment. Whilst performing the path, the agent of interest may encounter obstacles that it needs to avert. This would enable the search agent to perform an obstacle-free route which is required to be found before it starts its path to the destination. In a dynamic environment, where new obstacles may appear or existing obstacles may move in the workspace of the search agent, this process of finding an optimal or feasible route is repeated whenever new change in the environment is encountered. Hence, a need rises to construct a fast and efficient algorithm that can provide such functionality. The global path planning problem is known as an NP-complete problem, which led to creating several metaheuristic methods in order to solve this problem as fast and efficiently as possible, applied both in static and dynamic environments. Hybrid methods have also been 
proposed, which combined deterministic and metaheuristic techniques that provide optimal or feasible results, accelerating the speed of execution.

The workspace of the search agent can be easily discretized into smaller equal or not equal regions which can improve the manipulation and finding the optimal route to the destination point in the known environment. Each of these regions can be labelled as occupied, free or unknown, depending on the knowledge the agent has available about each of the regions or cells. Having this in mind, many gridbased approaches have been introduced throughout the past several years that divide the agent's workspace into equal regions. This provides sophisticated discretization which is utilized in a simple manner by the agent and can be further explored as such map, which again justifies the significance of this approach. Here, a clear separation of the concepts ought to be established between the local and global path planning. Whilst the local planner deals with the local obstacle avoidance, applying the needed velocities and angles of turning, the global planner intends to obtain a feasible or optimal collision-free path between the initial and destination point in the environment.

In the scope of this research, a new metaheuristic method for global path planning is presented. This technique, called the Quad Harmony Search (QHS) method, has been applied to the problem of global path planning for mobile robots in dynamic environments. It consists of two crucial phases. The first step is to perform a proper free space decomposition of the workspace by using the Quad-tree algorithm, and the second step is applying the metaheuristic Harmony Search method, a relatively new method which gained its popularity throughout the past few years. Then, the results from applying the new QHS technique in dynamic environments is elaborated.

\section{RELATED WORK}

The problem of path planning for mobile agents in dynamic environments has gradually increased its popularity in the past years. A great deal of the research studies published so far included utilization of optimization metaheuristic techniques and their various modifications by combining them with other deterministic and metaheuristic methods. Many of these publications used grid-based representations of the dynamic environment which justifies the need of applying these interpretations of the environment in practical applications. One such implementation was proposed by Lau et al. (2012) which efficiently discretizes the environment and obtains nearoptimal paths.

The Simulated Annealing (SA) algorithm is a well-known optimization technique that has been introduced in 1983. It is founded on the principles of slow cooling that appears in the annealing process in physics and is similar to the process of slow converging in the algorithm and considering more options (Aarts et a., 1997). The SA method has found its vast utilization in many practical applications. Ho and Liu (2010) presented a research study that created the path of the robot by using a Voronoi diagram. This information was then fed to the Dijkstra algorithm to obtain an obstacle-free route and then this data were used to compute the best path by implementing the SA algorithm, thus satisfying kinematic constraints. The evaluations proved that this approach performs better than the traditional SA algorithm. Miao and Huang (2012) proposed an enhanced SA technique which involved initial path selection as a heuristic and additionally included multiple mathematical operators. The enhanced SA algorithm has been tested and evaluated in various environments and it has been proven that it provided less computational costs, shorter execution time and simplicity and results have shown that it gives the best performance among other algorithms it has been compared to. In a later research by Miao and Tian (2013), another similar approach has been published which also involves enhanced SA algorithm. This new method included two additional mathematical operators as opposed to the previously described approach, and it involved tests and evaluations with both static and dynamic obstacles. It showed to improve the overall performance of the execution and obtain feasible or optimal solutions. This method was compared to the Dijkstra algorithm and proved to be applicable to real applications.

The Particle Swarm Optimization (PSO) algorithm is also an optimization technique that is widely known and is founded on mimicking the social behaviours of the individuals as a group. Therefore, this method emphasizes the social interactions and the group's abilities as opposed to the capabilities of the individuals (Eberhart and Kennedy, 1995). Raja and Pugazhenthi (2009) apply the PSO algorithm to path planning in dynamic environments. Various types of obstacles with different shapes have been taken into consideration, such as convex, concave and curved obstacles which had different velocities. Here, the valid particles are used in the population, which means only the valid obstacle-free paths are considered. This gives the opportunity to avoid the exploration of invalid particles and giving additional penalties to the fitness function. The proposed algorithm also provides the needed velocity of the robot depending on its length of the path. Nasrollahy and Javadi (2009) also published a research study that implemented the PSO algorithm in environments that contained both static and moving obstacles, and as an addition the target was also able to move itself in the robot's environment. The proposed method succeeded to provide improved time execution and also avoided local optima. Zhang et al. (2012) presented a PSO based multipleobjective algorithm for path planning in uncertain dynamic environments, which was mainly introduced to find a way to give the robot ability to avoid unexpected circumstances in its environment. Firstly, the degree of the risks in the environment is examined by implementing a membership function. Having this and the length of the path needed to be travelled, the problem could be simply formulated as constrained bi-objective optimization, and then the PSO algorithm was effectively applied. The method introduced various improvements to the algorithm to accelerate its 
execution, such as random based particle update, infeasible archive and constrained domination relationship. Results displayed the effectiveness of this approach by constructing Pareto optimal paths.

The Ant Colony Optimization (ACO) method is an optimization technique that is primarily founded on the bases of ants' behaviours and their instinctive process of finding food for their colonies (Dorigo and Di Caro, 1999). Garcia et al. (2009) proposed a modification of the traditional ACO algorithm and their novel approach was founded on the bases of Simple Ant Colony Optimization Meta-Heuristic (SACO-MH). The new algorithm, named SACOdm, where $\mathrm{d}$ is distance and $\mathrm{m}$ is memory, the ants are able to memorize their travelled paths and therefore the process of making decisions is dependent on the existing distance between the starting and the ending node. This alteration of the algorithm gave acceleration of 10 times in most of the examinations. The optimal route is retrieved by using the Fuzzy Inference System and is afterwards adjusted by utilizing the Simple Tuning Algorithm. This method was tested against static and dynamic obstacles and was applied to plain terrain environments. Duan et al. (2009) published a new Max-Min adaptive Ant Colony Optimization (ACO) method which was effectively implemented on coordinated trajectory re-planning for Multiple Uninhabited Aerial Vehicles (multi-UAVs), which is a very complex global path planning problem. Having the properties of multiUAVs in dynamic and unpredictable environments available as knowledge given a priori, the exploring abilities are improved by minimum and maximum pheromone trails in the ACO algorithm and the collisions between the UAVs are avoided by adopting the point pheromone. Having the possibility of collisions of these vehicles in the air and their simultaneous arriving in mind, at the beginning an Estimated Time of Arrival (ETA) ought to be established and then the speed and the path ought to be constructed. This approach has been tested in combat dynamic environments and the experimental results have proven that this approach is effective to be applied to practical applications.

The Genetic Algorithm (GA) is a method which was widely used in many practical optimization problems and is certainly one of the most exploited till recent days (Goldberg, 1989). This technique is based on the ideas of natural evolution and is therefore belonging to the category of evolutionary algorithms. Shi and Cui (2010) proposed an algorithm based on the GA and applied it to the problem of dynamic path planning in unknown environments for mobile agents which implemented navigation and avoiding collisions. The unique coding technique reduced the traditional complexity of the algorithm and accelerated the exploring performances by projecting data from two dimensions to one dimension, decreasing the search space needed to be explored. The obstacle-free route, the shortest path and the smoothness of the route are considered in the fitness function of the GA and the algorithm's efficiency is improved by selecting concrete genetic operators. This approach proved to obtain great efficiency in various dynamic environments. Kala et al. (2010) solves the global path planning problem with evolutionary algorithms by using two hierarchies. The coarser hierarchy obtains the optimal route in a static environment by utilizing the map of the environment. This map is then an object of reducing the resolution which greatly accelerates the execution. The finer hierarchy then utilizes a section of this map and the optimal route is obtained for both static and dynamic environments. These hierarchies are optimized during the execution of the route. The finer hierarchy also takes care of the obstacle avoidance of the search agent and assumes that all of the objects in the environment are moving in the same direction and have the same velocities. This approach provided high effectiveness both with static and moving obstacles.

\section{QUAD-HARMONY SEARCH ALGORITHM}

This research paper presents a novel Quad-Harmony Search (QHS) algorithm which is effectively applied to the global path-planning problem. The algorithm is composed of three main stages described with the diagram presented in Fig. 1.
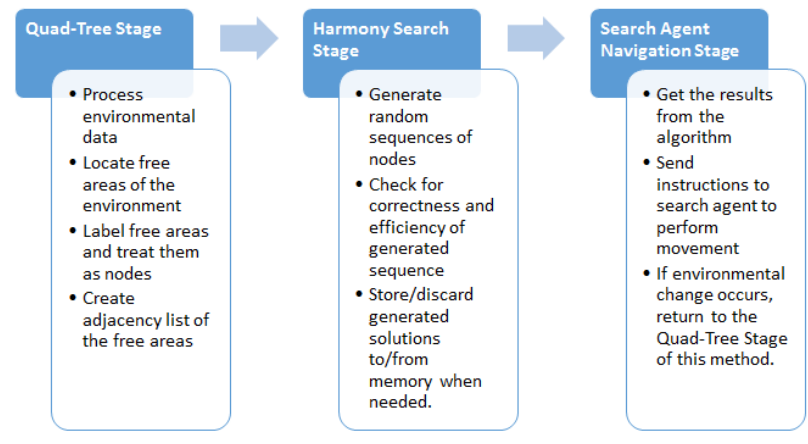

Figure 1 Main stages of the QHS algorithm

By using the Quad-tree free space decomposition algorithm, this algorithm splits the environmental data of the agent into four equally sized sub-grids in a recursive manner, until it finds a single cell or an obstacle-free sub-grid. In this phase, all of the free regions are labelled with proper numbers and treated as a single node later in the Harmony Search (HS) stage of the algorithm. The HS stage consists of applying the Harmony Search method (Geem et al., 2001) to find an optimal route, given the data provided from the Quad-tree stage of the algorithm. The search space of the agent is dynamic and the agent is able to move in four different directions, namely labelled as up, right, down and left.

The QT stage of the algorithm used for the QHS is executed in the following manner:

1. Give the necessary discretized environmental data to the mobile robot as input.

2. Check if there is a single cell. If this is the case, determine whether it's a free rectangle or an obstacle. If it's empty, label it with a unique number. Otherwise, label it with -1 . 
3. Separate the current examined part of the environment into four equally sized regions.

4. Explore these regions and check if they are obstacle-free.

a. When all of the regions are obstacle-free, label them with the same unique number.

b. When two adjacent regions are obstacle free, label them with the same unique number and repeat the recursion steps from Step 2.

c. When 4.a) and 4.b) result with a false outcome, repeat the recursion steps for all of the regions from Step 2.

The results from the QT stage of the algorithm are fed as input to the Harmony Search stage of the QHS algorithm. Here, the input is used to construct an adjacency list which should be used for the graph search of the algorithm. Then, these data are utilized for the purposes of the fitness function of the HS algorithm. The value of the fitness function is then computed as follows:

1. Initialization. Set the start node of the algorithm to the initial point of search of the mobile robot. Set this node as the current node.

2. Adjacent nodes search. Examine the current node and check for its label:

a. When the current node is the final node, terminate and exit with success. Otherwise, continue to step 2.b.

b. Examine the adjacent nodes of the current node. The next node to be selected is the node generated as the random value by the one present in the current value (member) of the candidate vector, modulus the number of neighbours the current node has. This number is the index of the node in the adjacency list of the current node and it belongs to the domain of numbers given by $[1$; maxRectangles], where maxRectangles is the largest unique number generated by the QT stage of the algorithm.

c. Set the randomly selected node as the current node. Increase the returning value of the fitness function by a predefined value. Repeat Step 2 for this particular node.

Hence, the mathematical formulation of the fitness function would be interpreted as follows:

$$
f(.)=\sum_{g \in G} \operatorname{NodeCost}(g)
$$

where $\mathrm{g}$ is the current examined node, $\mathrm{G}$ is the domain set of the $g$ node, and NodeCost $(g)$ stands for the cost of the $g$ node related to its position compared to the destination point, defined in the following manner:

$$
\operatorname{Node} \operatorname{Cost}(g)= \begin{cases}1, & g \text { is before the destination node } \\
0, & \begin{array}{l}
g \text { is a destination node, or } \\
\text { is after the destinationnode }
\end{array}\end{cases}
$$

\section{EVALUATION AND EXPERIMENTAL RESULTS}

The new QHS metaheuristic algorithm has been applied to the problem of path planning in dynamic environments and has been evaluated experimentally. The main aim of the experimental evaluation was to confirm the ability of generating optimal and collision free path of the developed algorithm and to compare it with other metaheuristic algorithms. For the experimental evaluation of the developed algorithm, specific laboratory setup for indoor motion planning was used.

In our experiments a three wheel mobile robot (Figure 2) which is a modified version of the ARobot (ARobot, http://www.arrickrobotics.com/arobot/) was used.

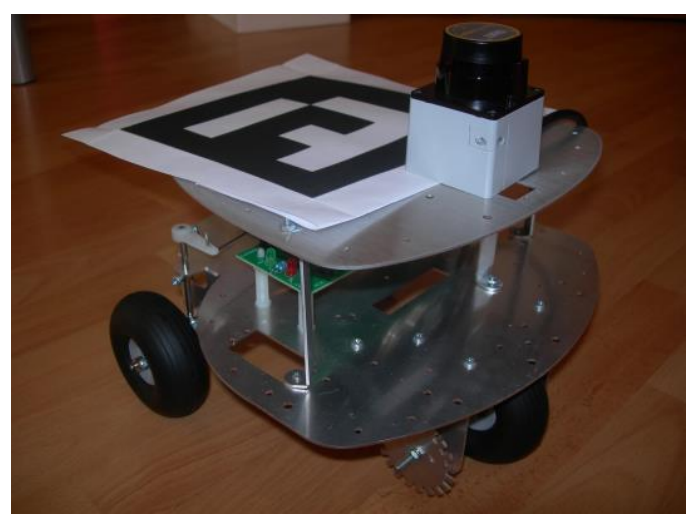

Figure 2 Robot used for experimental evaluation

The robot contains one Basic Stamp II controller from Paralax (Parallax, http://www.parallax.com), and two coprocessors: PIC16F84 for motor control. The robot has following sensors: sonar, two light sensors, temperature sensor, whisker sensors, PIR passive infrared motion detector, digital compass, R283-HOKUYO-LIDAR and sound output transducer. The robot has two 12 volt DC drive motors. These motors are regulated independently using PWM controlled H-bridges. The robot also has optical encoders that enable determination of speed and position of robot's wheels. All these components are placed in a lightweight aluminium construction with dimensions: 10 " $\mathrm{x}$ $10 ", 5 "$ tall and a payload capacity of 3lbs.

Mobile robot is connected to the control workstation via wireless link - using RF two-way radio modules. The workstation is running a program that is responsible for robot localization, path planning and motion control planning.

The architecture of the system designed for this experimental evaluation study is presented in Figure 3.

The sensors of the robot enabled it to gather data from the environment related to the state of the subspaces it currently visits. Initially, the environmental map of the workspace ought to be given to the mobile robot in order to perform its regular operations. During its traveling to the destination, the robot is capable of detecting changes in the workspace, such as new obstacles or obstacles in motion. This is 
performed in such way that the robot is able to sense the changes happening in 2 units (a unit is the size of one side of the cell) in every direction (north, south, east and west). If such transformations have happened in the environment, then the QHS algorithm is run one more time, but now having its current cell set as the starting cell of the map and the map is updated with the new modifications. The division of the search space is executed, the adjacency list of rectangular subspaces is recreated, and the path used for traveling to the destination is constructed again. This is a process that is repeated whenever a change occurs in the robot's workspace. Otherwise, if the map is not modified, the mobile robot executes the path without further examinations.

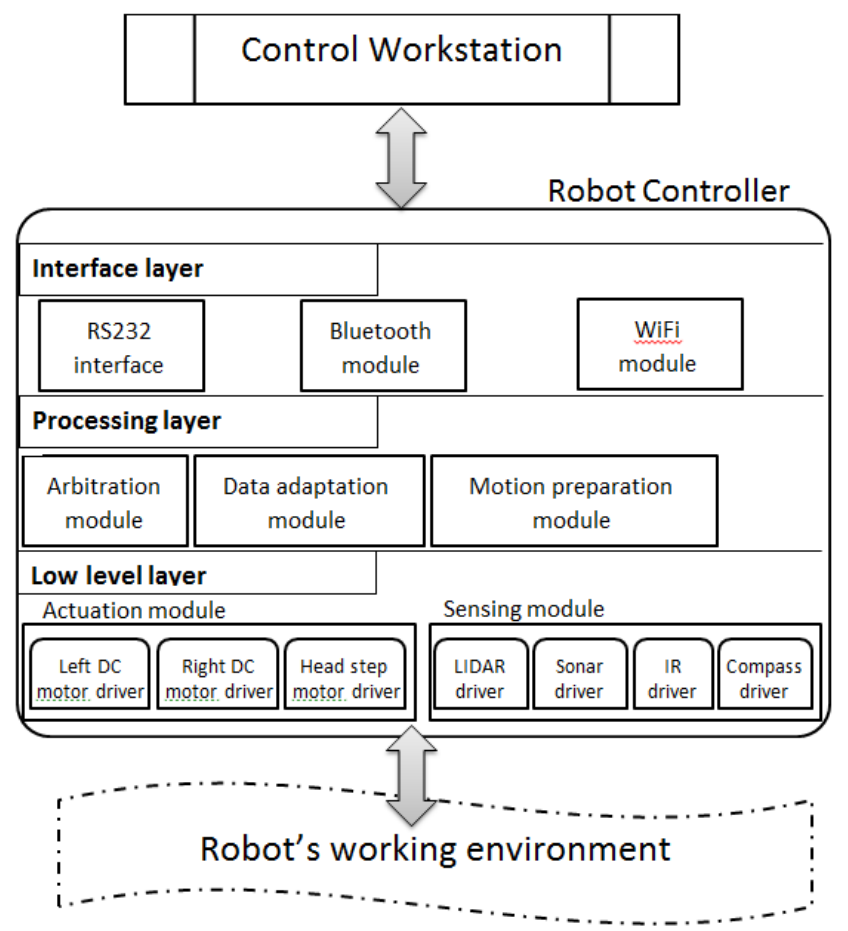

Figure 3 Architecture of the system designed for experimental evaluation

For the purposes of the research elaborated in this paper, different test cases having various percentages of obstacles and grid sizes have been taken into consideration. The examined grids had percentages of obstacles in range of $10 \%-90 \%$ and the grid sizes had dimensions of $8 \times 8,16 \times 16$, $32 \times 32,64 \times 64$ and $128 \times 128$. The new QHS metaheuristic algorithm has been compared to the Ant Colony (ACO), Genetic Algorithm (GA), Particle Swarm Optimization (PSO) and the Simulated Annealing (SA) algorithm.

The formula used to evaluate the performance of the QHS algorithm applied to dynamic environments uses the total time execution as main metrics, and is given as follows (3):
In the formula (3), s stands for a state of single modification of the map, $S$ stands for the set of changes occurring whilst executing the best path, and $T(s)$ stands for the time of performance of the algorithm happening in the state of change $s$.

The comparison of the above mentioned metaheuristic algorithms, i.e. QHS, AC, GA, PSO and SA algorithms for dynamic environments in terms of their execution time for grid sizes of $8 \times 8,16 \times 16,32 \times 32,64 \times 64$ and $128 \times 128$, respectively is presented in Fig. 4-8.

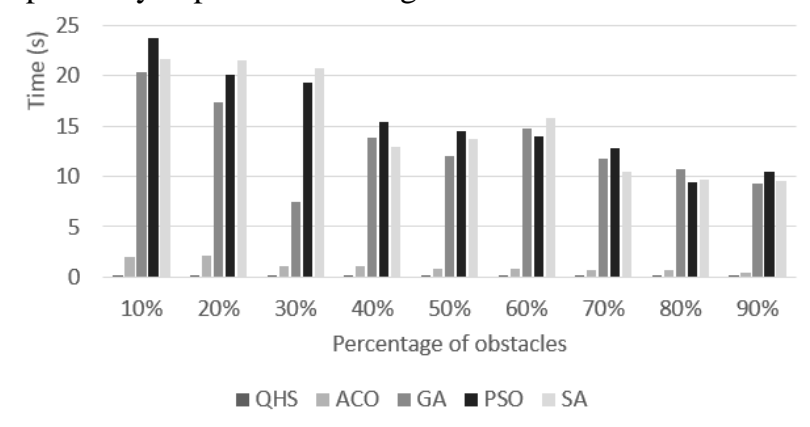

Figure 4 Results from examining dynamic environments for $8 x 8$ grid sizes.

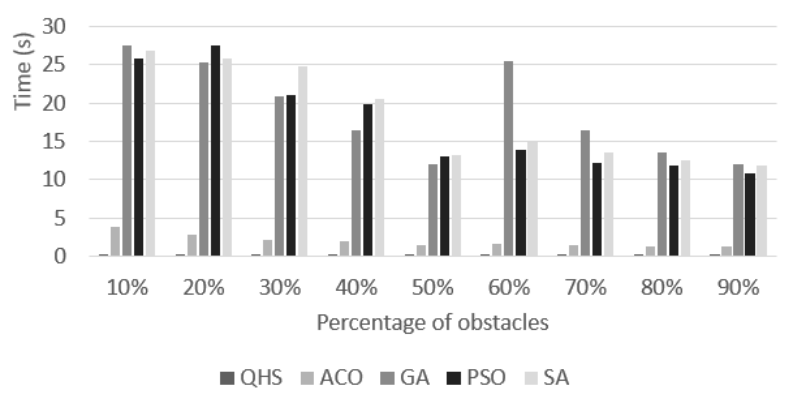

Figure 5 Results from examining dynamic environments for $16 \times 16$ grid sizes.

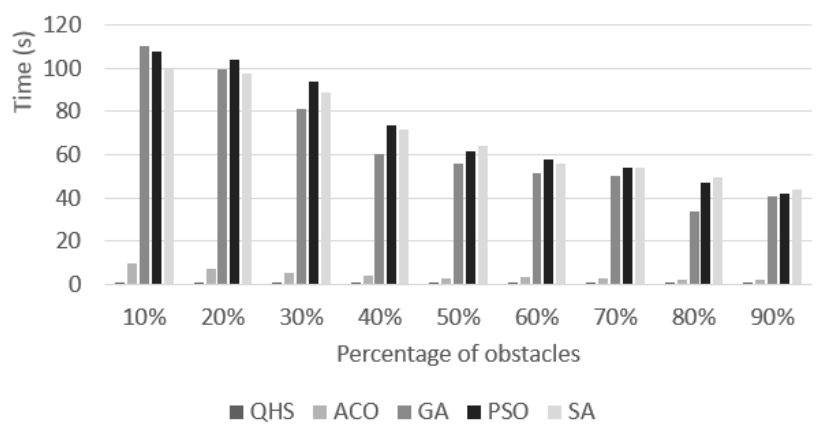

Figure 6 Results from examining dynamic environments for $32 \times 32$ grid sizes. 


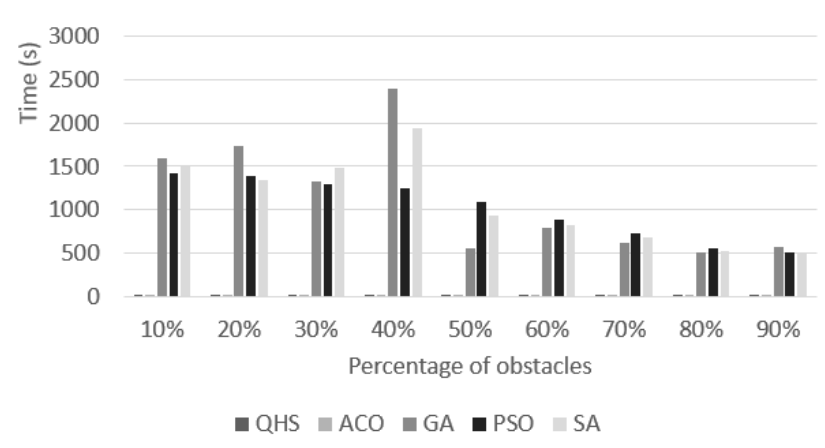

Figure 7 Results from examining dynamic environments for $64 x 64$ grid sizes.

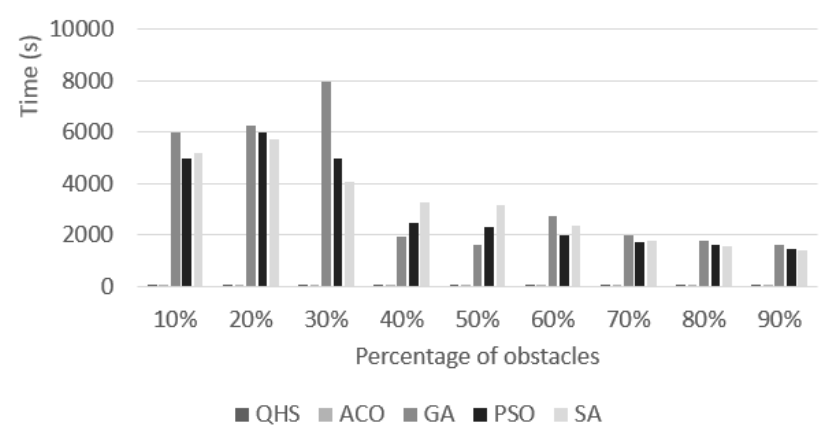

Figure 8 Results from examining dynamic environments for $128 \times 128$ grid sizes.

As can be seen, the GA, PSO and SA algorithms needed greater time of execution in order to obtain a feasible path from the starting point to the destination. Their time of execution varies and is mainly unpredictable in terms of various percentages of obstacles, hence the fluctuations that can be noticed from the Figures 4-8. The ACO algorithm performed much better than these algorithms and it followed a trend of gradual decrease following the increase of the number of obstacles. In the end, QHS gave the best results in terms of time, which is influenced by the division of the search space into smaller subspaces and creating an adjacency list of these neighbouring regions, which reduces the nodes that need to be explored. As it was expected, this algorithm needed more time to perform for the grids having between $30 \%$ and $60 \%$ of obstacles, since here the number of separations of the workspace is expectedly higher. In all of the test cases, QHS proved to give best results among the above mentioned metaheuristic algorithms.

The optimality of these results has been confirmed by using the deterministic Depth-First Iterative-Deepening (DFID) algorithm. The results in terms of lengths of the optimal paths are the same for QHS and DFID and they are given in Table 1.

Table 1 Optimal path lengths - equal for both QHS and DFID.

\begin{tabular}{|c|c|c|c|c|c|}
\hline \multirow{2}{*}{$\begin{array}{c}\text { Percentage } \\
\text { of } \\
\text { obstacles }\end{array}$} & \multicolumn{5}{|c|}{ Grid sizes } \\
\cline { 2 - 6 } & $\mathbf{8 x 8}$ & $\mathbf{1 6 x 1 6}$ & $\mathbf{3 2 x 3 2}$ & $\mathbf{6 4 x 6 4}$ & $\mathbf{1 2 8 x 1 2 8}$ \\
\hline $10 \%$ & 14 & 30 & 66 & 133 & 74 \\
\hline
\end{tabular}

\begin{tabular}{|c|c|c|c|c|c|}
\hline $20 \%$ & 14 & 30 & 61 & 114 & 71 \\
\hline $30 \%$ & 16 & 28 & 66 & 100 & 89 \\
\hline $40 \%$ & 13 & 42 & 50 & 234 & 66 \\
\hline $50 \%$ & 9 & 23 & 51 & 38 & 53 \\
\hline $60 \%$ & 6 & 13 & 19 & 22 & 30 \\
\hline $70 \%$ & 3 & 7 & 9 & 11 & 47 \\
\hline $80 \%$ & 6 & 10 & 5 & 8 & 28 \\
\hline $90 \%$ & 5 & 7 & 7 & 4 & 59 \\
\hline
\end{tabular}

The results from the DFID algorithm proved to be exactly the same as the lengths of the paths obtained by the newly presented QHS algorithm and by the ACO algorithm. As opposed to these results, the GA, PSO and SA algorithm never gave the optimal paths and were easily stuck in local optima, hence the greater time of execution to obtain feasible results. This proves once again that the QHS algorithm performs better in terms of time and optimality of the obtained solutions, which makes it a suitable solution that can be applied to the problem of path planning for mobile robots in dynamic environments.

\section{CONCLUSION AND FUTURE WORK}

This paper presented a novel metaheuristic approach to solving the global path planning problem in dynamic environments, namely the QHS algorithm. This algorithm was founded on the effective search space division capabilities of the Quad-tree algorithm and the optimization capabilities of the Harmony Search algorithm. Different grid sizes and different percentages of obstacles have been examined and experimental results have been elaborated. This research has shown that this algorithm performs better than other metaheuristic methods, namely the ACO, GA, PSO and SA algorithms in terms of time and acceleration of convergence. Hence, this algorithm is a great foundation for further researches in the field of metaheuristic algorithms applied to NP-complete problems, such as the global path planning problem detailed in this paper.

\section{REFERENCES}

Aarts, E. H., Korst, J. H., \& van Laarhoven, P. J. (1997). Simulated annealing, Local Search in Combinatorial Optimization (Emile Aarts and Jan Karel Lenstra, eds.).

Dorigo, M., \& Di Caro, G. (1999). Ant colony optimization: a new meta-heuristic. In Evolutionary Computation, 1999. CEC 99. Proceedings of the 1999 Congress on (Vol. 2). IEEE.

Duan, H. B., Zhang, X. Y., Wu, J., \& Ma, G. J. (2009). Max-min adaptive ant colony optimization approach to multi-UAVs coordinated trajectory replanning in dynamic and uncertain environments. Journal of Bionic Engineering, 6(2), 161-173.

Garcia, M. A., Montiel, O., Castillo, O., Sepúlveda, R., \& Melin, P. (2009). Path planning for autonomous mobile robot navigation with ant colony optimization and fuzzy cost function evaluation. Applied Soft Computing, 9(3), 11021110 . 
Geem, Z. W., Joong H. K., \& Loganathan, G. V. (2001). A new heuristic optimization algorithm: harmony search. Simulation 76.2 (2001): 60-68.

Goldberg, D. (1989). Genetic Algorithms in optimization, search and machine learning. Addison Wesley, New York.

Eberhart, R., \& Kennedy, J. (1995, October). A new optimizer using particle swarm theory. In Micro Machine and Human Science, 1995. MHS'95., Proceedings of the Sixth International Symposium on (pp. 39-43). IEEE.

Eiben AE, Smith JE (2003) Introduction to Evolutionary Computing. Springer.

Ho, Y. J., \& Liu, J. S. (2010, March). Simulated annealing based algorithm for smooth robot path planning with different kinematic constraints. In Proceedings of the 2010 ACM Symposium on Applied Computing (pp. 1277-1281). ACM.

Jacq J, Roux C (1995) Registration of non-segmented images using a genetic algorithm. Lecture notes in computer science, 905, 205-211.

Kala, R., Shukla, A., \& Tiwari, R. (2010). Dynamic environment robot path planning using hierarchical evolutionary algorithms. Cybernetics and Systems: An International Journal, 41(6), 435454.

Lau, B., Sprunk, C., \& Burgard, W. (2012). Efficient grid-based spatial representations for robot navigation in dynamic environments. Robotics and Autonomous Systems.

Miao, H., \& Huang, X. (2012). Dynamic vehicle path planning using an enhanced simulated annealing approach for supply chains. International Journal of Enterprise Network Management, 5(2), 197-218.

Miao, H., \& Tian, Y. C. (2013). Dynamic robot path planning using an enhanced simulated annealing approach. Applied Mathematics and Computation, 222, 420-437.

Nasrollahy, A. Z., \& Javadi, H. (2009, November). Using particle swarm optimization for robot path planning in dynamic environments with moving obstacles and target. In Computer Modeling and Simulation, 2009. EMS'09. Third UKSim European Symposium on (pp. 60-65). IEEE.

Raja, P., \& Pugazhenthi, S. (2009, October). Path planning for mobile robots in dynamic environments using particle swarm optimization. In Advances in Recent Technologies in Communication and Computing, 2009. ARTCom'09. International Conference on (pp. 401-405). IEEE.

Shi, P., \& Cui, Y. (2010, May). Dynamic path planning for mobile robot based on genetic algorithm in unknown environment. In Control and Decision Conference (CCDC), 2010 Chinese (pp. 4325-4329). IEEE.

Zhang, Y., Gong, D. W., \& Zhang, J. H. (2012). Robot path planning in uncertain environment using multi-objective particle swarm optimization. Neurocomputing.

\section{WEBSITES}

ARobot, http://www.arrickrobotics.com/arobot/.

Parallax, http://www.parallax.com. 